\title{
Neutrino Mass and Higgs Self-Coupling Predictions
}

\author{
Ole L. Trinhammer \\ Department of Physics, Technical University of Denmark, Kongens Lyngby, Denmark \\ Email: ole.trinhammer@fysik.dtu.dk
}

How to cite this paper: Trinhammer, O.L (2017) Neutrino Mass and Higgs SelfCoupling Predictions. Journal of Modern Physics, 8, 926-943.

https://doi.org/10.4236/jmp.2017.86059

Received: April 8, 2017

Accepted: May 24, 2017

Published: May 27, 2017

Copyright (C) 2017 by author and Scientific Research Publishing Inc. This work is licensed under the Creative Commons Attribution International License (CC BY 4.0).

http://creativecommons.org/licenses/by/4.0/

\begin{abstract}
Combining with cosmological constraints we find a most probable value of $17.6 \mathrm{meV}$ for beta decay anti-neutrinos. In passing we note that our expectation for the quadric Higgs self-coupling deviates from standard model expectations by a factor equal to the ud quark mixing matrix element. This matrix element also turns up by its square root in the expected triple self-coupling. We present neutrino mass eigenstates related to the neutron beta decay. In our first scenario we get $15.2 \mathrm{meV}$ for the lowest mass eigenstate, in the second we get $0.917 \mathrm{eV}$. The latter is to be covered by the KATRIN experiment, while the former comes close to the CRES sensitivity in the Project 8 reach.
\end{abstract}

\section{Keywords}

Neutrino Mass, Higgs Self-Coupling, Intrinsic Quantum Mechanics

\section{Introduction}

Observation of neutrino oscillations between the three lepton flavour species, $v_{\mathrm{e}}, v_{\mu}, v_{\tau}[1]$ [2], means that at least two different mass eigenstates have nonvanishing mass. In particular one may mention the disappearance of solar neutrinos $v_{\mathrm{e}}$ [3] as a first indication for transformation of flavour states together with $v_{\mu} \rightarrow v_{\tau}$ oscillation [4] [5] and $v_{\mu} \rightarrow v_{\mathrm{e}}$ oscillation [6] [7] as spectacular confirmations. From the oscillations one infers mass differences between mass eigenstates. Now the task remains to determine the masses themselves. An experimental set-up, KATRIN [8] [9] [10] using tritium decay is undertaken in Karlsruhe, Germany with results for electron-based neutrinos expected in $2018^{1}$.

${ }^{1}$ See the KATRIN homepage, http://www.kit.edu/kit/english/20624.php, where it reads: "Erste interessante Ergebnisse zur Neutrinomasse werden bereits für Mitte 2018 erwartet". And further: "Die endgültige geplante Sensitivität erreicht KATRIN aber erst nach fünf Kalenderjahren Messzeit”, kes. 17.10.2016. 
We suggest the electron-based anti-neutrino mass scale to originate in a slightly misaligned Higgs vacuum [11] [12] [13] [14] [15] occurring in the neutron beta decay

$$
n \rightarrow p+e+\overline{v_{\mathrm{e}}} .
$$

In our first scenario, we find

$$
\frac{m_{\bar{V}}}{m_{\mathrm{e}}}=\frac{\alpha_{\mathrm{Z}}}{8 \sin ^{2} \theta_{\mathrm{W}} \cos ^{2} \theta_{\mathrm{W}}}\left(\frac{\alpha_{\mathrm{e}}}{\pi}\right)^{2},
$$

which yields $m_{\bar{v}} c^{2}=15.152(4) \mathrm{meV}$. In our second scenario we find

$$
\frac{m_{\bar{V}}}{m_{\mathrm{e}}}=\frac{1}{\pi \mathrm{E}_{\mathrm{e}}} \frac{\alpha_{\mathrm{z}}}{8 \sin ^{2} \theta_{\mathrm{w}} \cos ^{2} \theta_{\mathrm{w}}} \frac{\alpha_{\mathrm{e}}}{\pi},
$$

with $\mathrm{E}_{\mathrm{e}}=2.2655 \cdots$, which yields $m_{\bar{V}} c^{2}=0.9165(4) \mathrm{eV}$. The latter is below the limit $2 \mathrm{eV}$ from tritium decay [2] but above the limit $m_{\mathrm{tot}} \leq 0.2 \mathrm{eV} / \mathrm{c}^{2}$ on the sum-total of stable neutrino masses from cosmological phenomenology [16].

The first scenario value is comparable in order of magnitude with [2]

$$
\begin{gathered}
\sqrt{\left|\Delta m_{21}^{2}\right|}=(8.68 \pm 0.10) \mathrm{meV} \\
\sqrt{\left|\Delta m_{32}^{2}\right|}=(49.4 \pm 0.6) \mathrm{meV} \text { (normal hierarchy) } \\
\sqrt{\left|\Delta m_{32}^{2}\right|}=(50.1 \pm 0.6) \mathrm{meV} \text { (inverted hierarchy) }
\end{gathered}
$$

determined from the observed neutrino oscillations. The first scenario value at $15 \mathrm{meV}$ positions itself intriguingly with respect to the Cyclotron Radiation Emission Spectroscopy technique of Project 8 [17]. Project 8 states a lower bound $m_{v_{\mathrm{e}}} \geq 9(0.1) \mathrm{meV}$ from neutrino oscillations, cf. the first equation in (4) and they expect their own sensitivity level to go down to $m_{v_{\mathrm{e}}} \leq 40 \mathrm{meV}$.

Neutrino oscillations are traditionally described by mixing between left handed flavour fields via a non-diagonal matrix $U$ relating to left handed mass eigenstates [18]

$$
v_{l \mathrm{~L}}(x)=U_{l j} v_{j \mathrm{~L}}(x), \quad l=e, \mu, \tau
$$

From the Pontecorvo-Maki-Nakagawa-Sakata mixing matrix $U$ one gets "effective" flavour masses. For the anti-neutrino state created in the beta decay, one has [19]

$$
\left\langle m_{\beta}^{2}\right\rangle=\sum_{j}\left|U_{e j}\right|^{2} m_{\bar{v}_{j}}^{2}
$$

where the sum runs over the mass eigenstates $\bar{v}_{j}$. It is not known whether $j=1,2, \cdots$ ends at $j=3$.

We write only $\bar{v}$ for the mass eigenstate in (2) and (3). In the discussion section we make a choice on hierarchy. For a three neutrino model in normal hierarchy we get from (6) our most probable mass value for the neutrino flavour generated in beta decay

$$
\sqrt{\left\langle m_{\beta}^{2}\right\rangle}=17.6 \pm 0.2 \mathrm{meV} / \mathrm{c}^{2}
$$




\section{Leptonic Sector}

Both our scenarios set out from an intrinsic description of the electron, related to a similar description of the nucleon.

The value $E_{e}$ in the second scenario (3) is found from an electronic ground state on the intrinsic configuration space, the Lie group $U(2)$ with a hamiltonian structure

$$
\Lambda_{\mathrm{e}}\left[-\frac{1}{2} \Delta+\frac{1}{2} \operatorname{Tr} \chi^{2}\right] \Psi(u)=\mathcal{E} \Psi(u)
$$

where $\Lambda_{\mathrm{e}}$ is the energy scale and $u=e^{i \chi} \in U(2)$ is the configuration variable. We assume the lowest neutrino mass eigenstate to be the ground state of a similar hamiltonian structure

$$
\Lambda_{v}\left[-\frac{1}{2} \Delta+\frac{1}{2} \operatorname{Tr} \chi^{2}\right] \Psi(u)=\mathcal{E} \Psi(u) .
$$

The two Equations (8) and (9) share dimensionless eigenvalues for the ground state, i.e.

$$
\mathrm{E}_{\mathrm{e}} \equiv \mathcal{E}_{\mathrm{e}} / \Lambda_{\mathrm{e}}=\mathcal{E}_{v} / \Lambda_{v} \equiv \mathrm{E}_{v},
$$

where $\mathcal{E}_{\mathrm{e}}=m_{\mathrm{e}} c^{2}$ and $\mathcal{E}_{v}=m_{v} c^{2}$.

If we can solve (8) (and we can), all that is needed to determine $m_{v}$ is to fix $\Lambda_{v}$. Below we shall present two different scenarios for the determination of $\Lambda_{v}$.

\section{The Leptonic Ground State}

The particle data group notes that existing upper limits on neutrino masses imply very low masses of the order of one millionth of charged lepton $m_{l}$ and quark masses $m_{q}$

$$
m_{j} / m_{l, q} \leq 10^{-6}
$$

and they conclude [20]: "It is natural to suppose that the remarkable smallness of neutrino masses is related to the existence of a new fundamental mass scale in particle physics and thus to new physics beyond that predicted by the Standard Model".

In the present work we suggest two mass scale scenarios. The new physics component offered in that connection is the idea of intrinsic configuration variables.

The configuration variables in (8) and (9) contain four dynamical variables from the four dimensions laid out by the four generators of $U(2)$. Two of the generators are toroidal, i.e. diagonal $2 \times 2$ matrices in a two-dimensional representation. We thus write

$$
u=e^{i\left(\vartheta_{1} T_{1}+\vartheta_{2} T_{2}+\alpha_{1} \sigma_{1}+\alpha_{2} \sigma_{2}\right)}, \quad \vartheta_{1}, \vartheta_{2}, \alpha_{1}, \alpha_{2} \in \mathbb{R},
$$

where $\sigma_{1}, \sigma_{2}$ are the two off-diagonal Pauli matrices

$$
\sigma_{1}=\left\{\begin{array}{ll}
0 & 1 \\
1 & 0
\end{array}\right\}, \sigma_{2}=\left\{\begin{array}{cc}
0 & -i \\
i & 0
\end{array}\right\}
$$


and the two diagonal generators

$$
T_{j}=-i \frac{\partial}{\partial \vartheta_{j}}
$$

are represented by

$$
T_{1}=\left\{\begin{array}{ll}
1 & 0 \\
0 & 0
\end{array}\right\}, T_{2}=\left\{\begin{array}{ll}
0 & 0 \\
0 & 1
\end{array}\right\} .
$$

The more common parametrization from using

$$
\mathbf{1}=\left\{\begin{array}{ll}
1 & 0 \\
0 & 1
\end{array}\right\}, \sigma_{3}=\left\{\begin{array}{cc}
1 & 0 \\
0 & -1
\end{array}\right\}
$$

as diagonal $U(2)$ generators is equivalent to the choice in (15) but does not match the polar decomposition of the Laplacian $\Delta$ which we need in order to solve (8) and (9).

The wavefunction in (8) can be factorized in a torodial and an off-torus part

$$
\Psi(u)=\tau\left(\vartheta_{1}, \vartheta_{2}\right) \Upsilon\left(\alpha_{1}, \alpha_{2}\right) .
$$

in analogy with solving the Hydrogen atom in polar coordinates. The offtoroidal degrees of freedom can be integrated out to get for the measure-scaled toroidal wavefunction $R=J \tau$

$$
\left[-\frac{1}{2}\left(\frac{\partial^{2}}{\partial \vartheta_{1}^{2}}+\frac{\partial^{2}}{\partial \vartheta_{2}^{2}}\right)+W\left(\vartheta_{1}, \vartheta_{2}\right)\right] R\left(\vartheta_{1}, \vartheta_{2}\right)=\operatorname{ER}\left(\vartheta_{1}, \vartheta_{2}\right)
$$

with the van de Monde determinant [21]

$$
J=2 \sin \frac{1}{2}\left(\vartheta_{1}-\vartheta_{2}\right)
$$

and with potential

$$
W\left(\vartheta_{1}, \vartheta_{2}\right)=-\frac{1}{4}+\frac{1}{16} \frac{s(s+1)-s^{2}}{\sin ^{2} \frac{1}{2}\left(\vartheta_{1}-\vartheta_{2}\right)}+w\left(\vartheta_{1}\right)+w\left(\vartheta_{2}\right) .
$$

Here the trace potential from (8) spells out as, see Figure 1

$$
\frac{1}{2} \operatorname{Tr} \chi^{2}=w\left(\vartheta_{1}\right)+w\left(\vartheta_{2}\right)
$$

with periodic parametric potentials [22]

$$
w(\vartheta)=\frac{1}{2}(\vartheta-n \cdot 2 \pi)^{2}, \vartheta \in[(2 n-1) \pi,(2 n+1) \pi], n \in \mathbb{Z} .
$$

and the nominator $s(s+1)-s^{2}$ in the centrifugal term is obtained by using $\sigma_{1}^{2}+\sigma_{2}^{2}=\sigma^{2}-\sigma_{3}^{2}$ for states of spin $s$. The constant curvature [23] term $1 / 4$ and the centrifugal term originate in the Laplacian [24]

$$
\Delta=\sum_{j=1}^{2} \frac{1}{J^{2}} \frac{\partial}{\partial \vartheta_{j}} J^{2} \frac{\partial}{\partial \vartheta_{j}}-\frac{1}{J^{2}} \frac{\sigma_{1}^{2}+\sigma_{2}^{2}}{2},
$$

where $\vartheta_{j}$ are dynamical toroidal eigenangles from the two eigenvalues $e^{i \vartheta_{j}}$ of the configuration variable $u$.

The eigenvalue of the ground state in (8), respectively (18), can be lowered by 
allowing period doublings in the measure-scaled torodial wavefunction, see Figure 2. This is possible because of the periodic nature of the potential which opens for Bloch degrees of freedom like in solid state physics [25]. In order that the wavefunction remains single-valued on $U(2)$ the Bloch phase factors are restricted to $e^{i \kappa \theta}$ with $\kappa=0, \pm \frac{1}{2}$. For $4 \pi$-periodic states we expand $R$ in (18) on Slater determinants [26]

$$
f_{p q}-f_{q p}=\left|\begin{array}{ll}
e^{i p \vartheta_{1}} & e^{i p \vartheta_{2}} \\
e^{i q \vartheta_{1}} & e^{i q \vartheta_{2}}
\end{array}\right|
$$

with half odd-integer $p, q$.

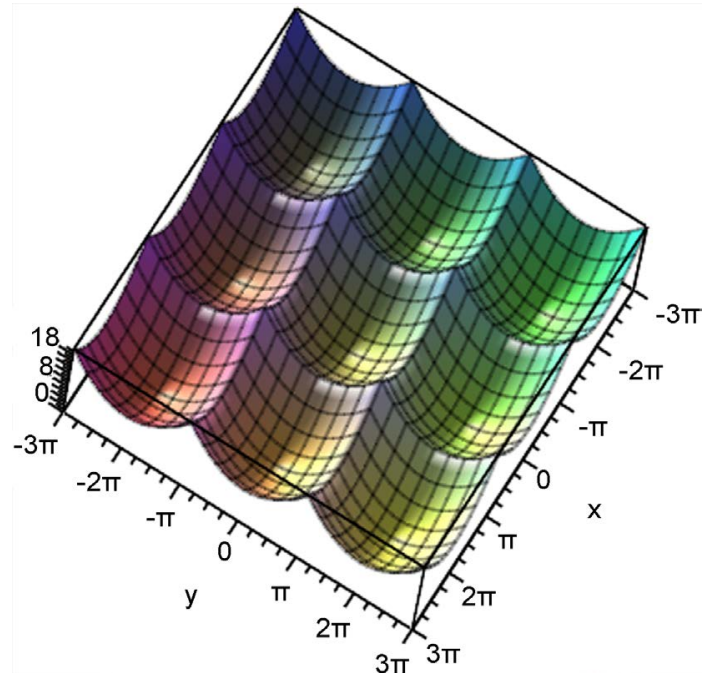

Figure 1. Parametric periodic potential, "egg-tray”. The periodicity in coordinate space represents the compact nature of the intrinsic configuration space of our description. The colour shading is only to enhance the $3 \mathrm{D}$ perception.

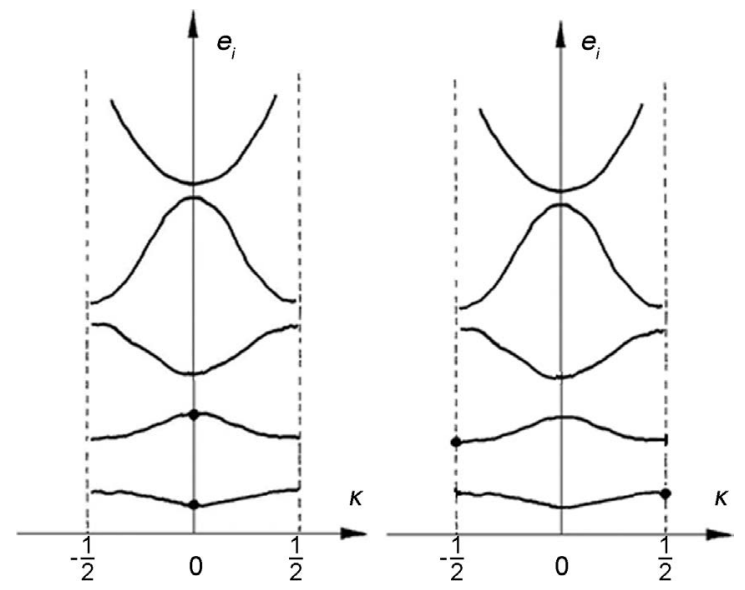

Figure 2. Reduced zone scheme [25] for the one-dimensional Equation (25). The period doubling in the diminished state for level two is paired with an augmented period doubled state for level one. The Bloch phase factors $e^{i \kappa \theta}$ with $\kappa= \pm \frac{1}{2}$ are chosen to compensate for those in the neutron to proton transition in Figure 4. The variation with $\kappa$ for the lowest levels is exaggerated for clarity. 
The electron rest energy $m_{\mathrm{e}} c^{2}$ is identified with the eigenvalue of the ground state $\mathcal{E}_{\mathrm{e}}=\mathrm{E}_{\mathrm{e}} \Lambda_{\mathrm{e}}$ in (8). In other words, $\mathrm{E}_{\mathrm{e}}$ is the dimensionless eigenvalue. We have determined $E_{e}=2.2655 \cdots$ by a Rayleigh-Ritz method [27] with 3368 base functions of the type (24) which is at the limit of our computer programme. All the integrals needed in the Rayleigh-Ritz procedure can be solved analytically (see appendix $C$ in [28] for a similar problem) which means that $E_{e}$ can be determined with high accuracy. The potential (21) is shown in Figure 1 with a characteristic periodic structure originating in mapping to a real parameter space from the compact configuration space. An alternative basis to (24) can be constructed as Slater determinants from solutions to the one-dimensional equation

$$
\left[-\frac{1}{2} \frac{\partial^{2}}{\partial \vartheta^{2}}+w(\vartheta)\right] \varphi_{i}(\vartheta)=e_{i} \varphi_{i}(\vartheta) .
$$

The two basis sets are both complete and yield the same spectrum as they should, but the integrals for the Rayleigh-Ritz solution based on parametric eigenfunctions from (25) can only be solved numerically. The reduced zone scheme for the lowest levels of (25) is shown in Figure 2. See e.g. [28] for more details on the parametric solutions.

\section{Baryonic Sector}

We have described the baryon spectrum by configurations on the Lie group $U$ (3) with dynamics determined by a Kogut-Susskind-inspired structure [29] [30]

$$
\frac{\hbar c}{a}\left[-\frac{1}{2} \Delta+\frac{1}{2} \operatorname{Tr} \chi^{2}\right] \Psi(u)=\mathcal{E} \Psi(u) .
$$

Here the configuration variable $u=e^{i \chi} \in U(3)$ and $\Lambda=\hbar c / a \approx 214 \mathrm{MeV}$ is the energy scale corresponding to a length scale $a$ which we took to be related to the classical electron radius $r_{\mathrm{e}}=e^{2} /\left(4 \pi \epsilon_{0} m_{\mathrm{e}} c^{2}\right)$ [31] [32] by the projective relation [30], see Figure 3

$$
\pi a=r_{\mathrm{e}} .
$$

This scale reproduces accurately the electron to neutron mass ratio

$$
\frac{m_{\mathrm{e}}}{m_{\mathrm{n}}}=\frac{\alpha\left(m_{\mathrm{n}}\right)}{\pi} \frac{1}{\mathrm{E}_{\mathrm{n}}} \approx \frac{1}{1838.9 \cdots}
$$

with the dimensionless eigenvalue $\mathrm{E}_{\mathrm{n}}=\mathcal{E}_{\mathrm{n}} / \Lambda \approx 4.38$ determined by solving (26). It also reproduces the $\mathrm{N}$ and $\Delta$ baryon spectrum with no missing resonance problem [33]. Flavour degrees of freedom are hidden in the Laplacian [24]

$$
\Delta=\sum_{j=1}^{3} \frac{1}{J^{2}} \frac{\partial}{\partial \theta_{j}} J^{2} \frac{\partial}{\partial \theta_{j}}-\frac{1}{\hbar^{2}} \sum_{i<j, k \neq i, j} \frac{S_{k}^{2}+M_{k}^{2}}{8 \sin ^{2} \frac{1}{2}\left(\theta_{i}-\theta_{j}\right)},
$$

where [21] 


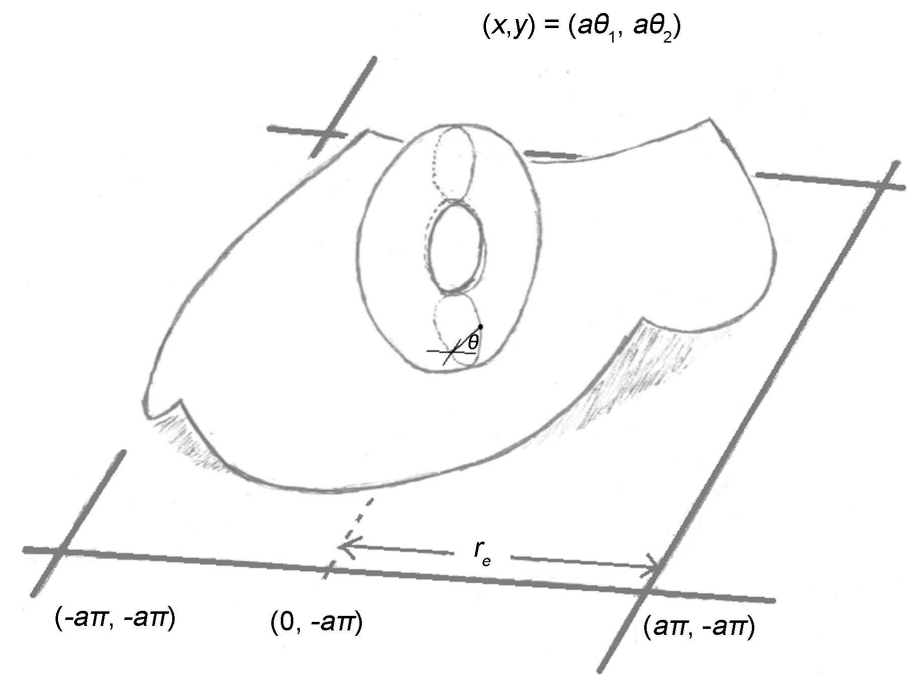

Figure 3. A projection from two of the three toroidal degrees of freedom of the intrinsic $U(3)$ configuration space used in our description of the neutron transformation in the neutron decay. We use the classical electron radius $r_{\mathrm{e}}$ [31] [32] to set the length scale $a$ for the projection of the intrinsic nucleonic dynamics as defined in (35). The thin line, small upper oval indicates a cut in the 2-dimensional surface representing the $U(2)$ torus which is outlined by the thicker line ovals in the center of the drawing. An orthogonal cut runs along an inner circle of the torodial surface. The lowest thin oval indicates the definition of toroidal angles $\theta$ used as intrinsic coordinates on the Lie group configuration space. After the cut, the toroidal surface maps onto a square in space. The curved sheet shows an intermediate step in the mapping. The inverse map, from laboratory space to intrinsic space is undertaken by the exponential mapping. See also Equation (12) for the leptonic case. The compact nature of the configuration space is manifested by periodicity in the projection space-think of a floor with square tiles. If the tiles are sheets made of rubber, you can fold the rubber sheet into a cylinder and sew the ends of the cylinder together to have a torus.

$$
J=\prod_{i<j}^{3} 2 \sin \frac{1}{2}\left(\theta_{i}-\theta_{j}\right)
$$

and the off-diagonal generators fulfil

$$
\left[M_{k}, M_{l}\right]=\left[S_{k}, S_{l}\right]=-i \hbar \epsilon_{k l m} S_{m} .
$$

Colour degrees of freedom are generated by the three $\frac{\partial}{\partial \theta_{j}} \mathrm{~s}$, intrinsic spin degrees of freedom are generated by the three $S_{k} \mathrm{~s}$ with commutators like body-fixed coordinate angular momenta in nuclear physics [34]. The flavour degrees of freedom are contained in the three $M_{k}$ s which connect the algebra. These mixing generators have a spectrum for $\mathbf{M}^{2}=M_{1}^{2}+M_{2}^{2}+M_{3}^{2}$ determined by the hypercharge quantum number $y$ and the isospin three component quantum number $i_{3} \quad[30]$

$$
\begin{gathered}
M^{2} / \hbar^{2}=\frac{4}{3}\left(n+\frac{3}{2}\right)^{2}-s(s+1)-3-\frac{1}{3} y^{2}-4 i_{3}^{3}, \\
n=0,1,2, \cdots
\end{gathered}
$$

together with the spin quantum number $s$ and the integer quantum number $n$ 
which can be thought of as an intrinsic toroidal excitation number analogous to the radial quantum number in the case of the hydrogen atom in a polar decomposition like (29)

$$
\Delta_{e, \text { polar }}=\frac{1}{r^{2}} \frac{\partial}{\partial r} r^{2} \frac{\partial}{\partial r}-\frac{1}{r^{2}} \mathbf{L}^{2}
$$

where $\mathbf{L}$ is the angular momentum operator. Compare (33) and (29) for the term "polar decomposition".

\section{Electroweak Scale-The Higgs Connection}

The transition from neutron to proton in (26) follows from a topological change in the wavefunction $\Psi$. For the neutronic state the wavefunction has a $2 \pi$-periodicity when parametrized in the toroidal eigenangles $\theta$ belonging to the three eigenvalues $e^{i \theta_{j}}$ of $u$ whereas for the protonic state, the wavefunction has a slightly broken symmetry with respect to the potential, namely a $4 \pi$-periodicity expressed by the appearance of fractional Bloch phase factors $e^{i \theta_{j} / 2}$ in the wavefunction. Such factors are allowed since the square of the wavefunction remains single-valued when extracted in parameter space, see also Bohr and Mottelson [35] for a note on doubling the angular domain for odd integer D-functions. The Bloch phase factors can lower the ground state eigenvalue-provided a mechanism exists to open the relevant degrees of freedom. That mechanism is the Higgs mechanism and we connected the strong and electroweak sectors by the Ansatz [33]

$$
\Lambda \theta=\alpha \varphi
$$

with strong energy scale $\Lambda$, torodial colour angle field $\theta$, electroweak fine structure coupling $\alpha$ and Higgs field $\varphi$.

We can support this Ansatz by old time quantum mechanics arguments based on the minimum quantum of action, $h$. The length scale a introduced above can be used for a space projection

$$
x_{j}=a \theta_{j}
$$

and a time projection [36]

$$
i c t=a \theta_{0} .
$$

A full shift of $2 \pi$ in the angular time variable $-i \theta_{0}$ corresponds to an exterior period $\tau$ determined by

$$
c \tau=a 2 \pi
$$

leading to a minimum action

$$
\Lambda \tau=h .
$$

As Planck's constant $h$ is the minimum unit of action in the time-domain, we can use $h c$ as a minimum unit of "action" in the space-domain, i.e. we have a minimum space action

$$
\Lambda \tau c=h c .
$$

For the exchange with the Higgs field we need a measure for the level of 
interaction energy. We take it to be $\alpha \varphi_{0}$, i.e. the electroweak fine structure coupling $\alpha$ times the vacuum expectation value $\varphi_{0}$ of the Higgs field. Thus the minimum space action to determine $\varphi_{0}$ is

$$
\alpha \varphi_{0} a=h c \text {. }
$$

Equating (39) and (40) we get

$$
\alpha \varphi_{0} a=\Lambda \tau c
$$

With the time period $\tau=2 \pi a / c$ from (37) and $\Lambda=\frac{\hbar c}{a}$ as in (26) we then have

$$
\varphi_{0}=\frac{2 \pi}{\alpha} \frac{h c}{a 2 \pi}=\frac{2 \pi}{\alpha} \Lambda
$$

This settles the electroweak scale $v$ by

$$
v / \sqrt{2}=\varphi_{0}
$$

corresponding to the standard model value [2] [33]

$$
v_{\mathrm{SM}}=246.22 \mathrm{GeV} \approx v \sqrt{V_{\mathrm{ud}}}=246.85 \mathrm{GeV} \text {. }
$$

See also arguments leading up to (58).

The $2 \pi$-shift behind (42) is what is needed for the topological change leading to the period doubling in the nucleonic wavefunction, see Figure 4 . In this figure, the Higgs potential

$$
V_{\mathrm{H}}(\phi)=\frac{1}{2} \delta^{2} \varphi_{0}^{2}-\frac{1}{2} \mu^{2} \phi^{2}+\frac{\lambda^{2}}{4} \phi^{4}
$$

mimics the parametrized intrinsic potential. The $2 \pi$-shift in $\theta$ is accompanied

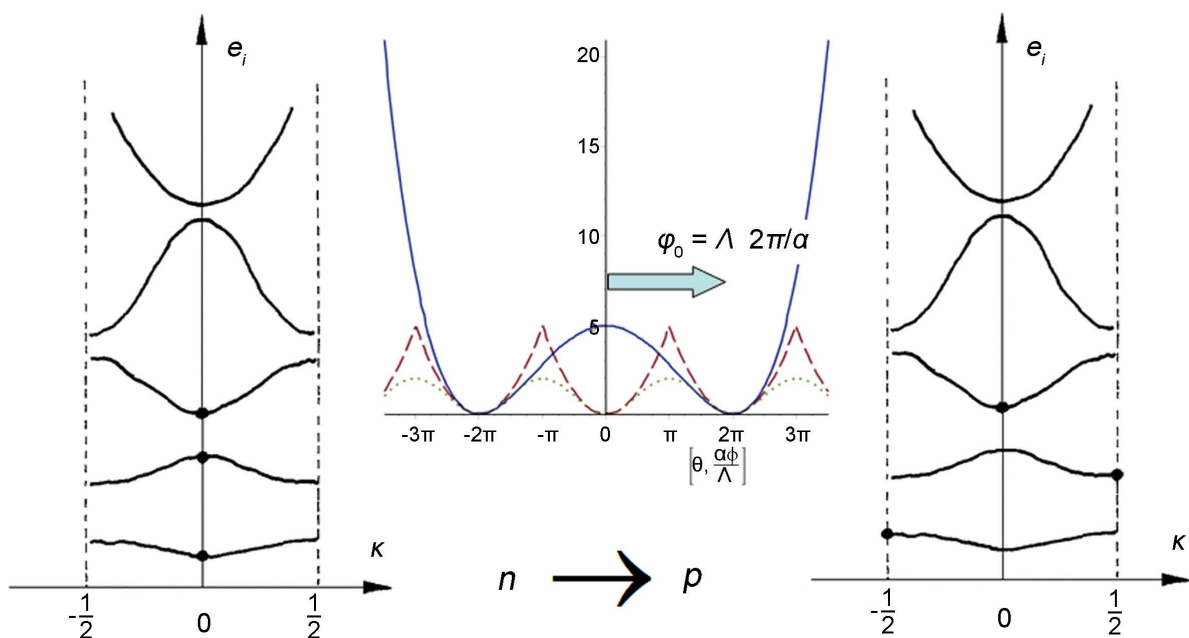

Figure 4. Higgs mechanism in neutron decay. The higgs potential (solid, blue) is structured by the intrinsic potential, either Wilson-inspired [37] (dotted, green) or Manton-inspired [38] (dashed, red), which are periodic in parameter space. Both parametric potentials yield the same value for the electroweak energy scale $v$, the Higgs mass $m_{\mathrm{H}}$ and the quadric Higgs self-coupling $\lambda^{2} / 4$ but only the Manton-inspired potential yields a satisfactory description of the baryon spectrum. The Manton-inspired potential expresses the euclidean measure folded onto the intrinsic configuration space [39]. Figure adapted from [40]. 
by a shift in the Higgs field from $\langle\phi\rangle=0$ before the neutron decay to $\langle\phi\rangle=\varphi_{0}$ after the neutron decay, see Figure 4 . The parameters in the Higgs potential are

$$
\frac{1}{2} \delta^{2}=\frac{1}{8} \varphi_{0}^{2}, \quad \mu^{2}=\frac{1}{2} \varphi_{0}^{2}, \quad \lambda^{2}=\frac{1}{2} .
$$

We revived the pionic Goldstone modes [41] by a slight vacuum misalignment in the Higgs mechanism with a misalignment angle $\zeta$ determined by (see Figure 5)

$$
\sin \zeta=\frac{\Lambda_{\mathrm{e}}}{\Lambda}
$$

With the mass parameter $\mu^{2}=\frac{1}{2} \varphi_{0}^{2}$ in the Higgs potential (45) we obtained the Higgs and pion masses determined by

$$
m_{\mathrm{H}} c^{2}=\frac{1}{\sqrt{2}} \varphi_{0} \cos \zeta=125.086( \pm 0.017) \mathrm{GeV}
$$

and

$$
m_{\pi} c^{2}=\frac{1}{\sqrt{2}} \varphi_{0} \sin \zeta=137.5 \mathrm{MeV}
$$

by using the trailing

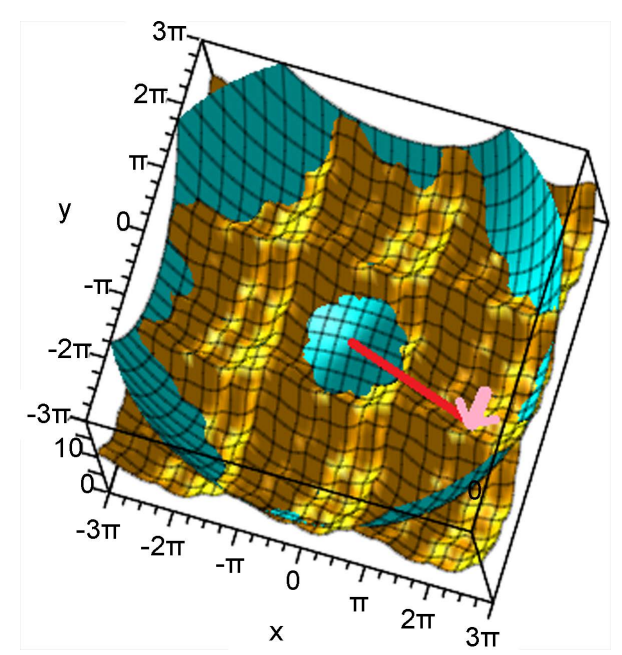

Figure 5. The Higgs potential (cyan) as a wine bottle bottom on a periodically rippled egg tray (orange). The egg-tray structure is the periodic parametric potential scaled from the baryonic sector and the ripples are scaled from the leptonic sector. Both are active in the neutron decay where the neutron changes to a charged proton and a chargecompensating electron. The size of the ripples is grossly exaggerated for clarity (drawing for $\sin \zeta=1 / 3$ as opposed the physical case $\sin \zeta \approx 1 / 1000$ in (47)). The size of the Higgs field vacuum expectation value $\varphi_{0}$ in (42) is shown by the red line. A component of the misalignment vector is shown as a rose arrow. The misalignment means that the toroidal coordinates $\left(\vartheta_{1}, \vartheta_{2}\right)$ in the leptonic sector are slightly rotated with respect to the toroidal coordinates $\left(\theta_{1}, \theta_{2}\right)$ in the baryonic sector, i.e. the ripples run slightly askew to the major structure. The misalignment even means a slight rotation into the third toroidal coordinate $\theta_{3}$ of the baryonic sector. This is not shown in the figure. Figure and revised caption from [41]. 


$$
2 \pi \Lambda=\alpha \varphi_{0}
$$

from (42). The value for the pion mass can be improved by iterative determination of the fine structure constant towards the pion mass scale.

We regret not seeing the connection (50) prior to the observation of the Higgs particle since it leads to a rather accurate value for the Higgs mass, see (48). Note however, that (46) also gives a prediction for the quadric Higgs self coupling

$$
\frac{g_{\text {hhhh }}}{4 !}=\frac{\lambda^{2}}{4}=\frac{1}{8}=0.125
$$

which still remains to be tested by experiment together with our prediction [41]

$$
\frac{g_{h h h}}{3 !}=\frac{\lambda^{2}}{4} v \cos \zeta=31.272(4) \mathrm{GeV}
$$

for the triple Higgs self-coupling. We here used the terminology of [42] for the mass and self-coupling terms of the Higgs particle field $h$

$$
\Delta L=-\frac{m_{h}^{2} c^{4}}{2} h^{2}-\frac{g_{h h h}}{3 !} h^{3}-\frac{g_{h h h h}}{4 !} h^{4}
$$

expanded about a minimum of the Higgs potential [42]

$$
V=-\frac{m_{h}^{2} c^{4}}{2} \phi^{2}+\lambda_{\mathrm{SM}} \phi^{4}
$$

The standard model values are [42]

$$
g_{h h h, \mathrm{SM}}=\frac{3 m_{h}^{2} c^{4}}{v_{\mathrm{SM}}} \text { and } g_{\text {hhh }, \mathrm{SM}}=\frac{3 m_{h}^{2} c^{4}}{v_{\mathrm{SM}}^{2}}
$$

where $v_{\mathrm{SM}}$ is the electroweak energy scale. The latter value is traditionally estimated from the Fermi constant in muon decay as [43]

$$
v_{\mathrm{SM}}=\sqrt{\frac{(\hbar c)^{3}}{\sqrt{2} G_{F \mu}}}=246.21965 \pm 0.00006 \mathrm{GeV} .
$$

On the other hand, from the vacuum expectation value $\varphi_{0}=v_{\mathrm{SM}} / \sqrt{2}$ at the minimum of the Higgs potential (54) one has

$$
v_{\mathrm{SM}}^{2}=\frac{m_{h}^{2}}{2 \lambda_{\mathrm{SM}}} .
$$

Since our $v$ is based on the $d$ to $u$ quark transformation in the $n$ to $p$ decay, $G_{F \beta}=G_{F \mu} V_{u d}$ [44] is used, i.e. a quark mixing matrix element $V_{u d}$ is introduced

$$
v_{\mathrm{SM}}=v \sqrt{V_{u d}} \text {. }
$$

In total we get for Higgs self-couplings relative to the standard model

$$
\begin{gathered}
\kappa_{h h h h} \equiv \frac{g_{h h h h}}{g_{h h h, \mathrm{SM}}}=\frac{\lambda^{2} / 4}{\lambda_{\mathrm{SM}}}=\frac{1 / 8}{m_{h}^{2} c^{4} /\left(2 v_{\mathrm{SM}}^{2}\right)}=V_{u d} \\
\kappa_{\text {hhh }} \equiv \frac{g_{h h h}}{g_{h h h, \mathrm{SM}}}=\frac{3 ! \cdot \frac{1}{8} \nu \cos \zeta}{3 m_{h}^{2} c^{4} / v_{\mathrm{SM}}}=\sqrt{V_{u d}} \cos \zeta \approx \sqrt{V_{u d}}
\end{gathered}
$$

which yields $\kappa_{h h h h}=0.97425(22)[2]$ and $\kappa_{h h h}=0.98704(11)$. Finally note that 
we actually announced the value $125.1 \mathrm{GeV}$ for the Higgs mass [45] almost a year prior to the result $125.09 \mathrm{GeV}$ from the combined ATLAS and CMS data [46]. Figure 6 shows the chronology.

\section{Neutrino Mass Scenario II}

The scale $\Lambda_{\mathrm{e}}$ in the leptonic sector is defined by the electron rest energy in (8) as $\Lambda_{\mathrm{e}} \equiv \mathcal{E}_{\mathrm{e}} / \mathrm{E}_{\mathrm{e}}=m_{\mathrm{e}} c^{2} / \mathrm{E}_{\mathrm{e}}$ with the dimensionless eigenvalue $\mathrm{E}_{\mathrm{e}}$ of the period-doubled ground state of (18). Conservation of spin in the neutron decay requires the creation of the anti-electron neutrino. Thereby also the idea of lepton number conservation is introduced. As the (anti)-neutrino has no charge, we assume its creation to be mediated by neutral weak currents, i.e. the coupling $\alpha$ is replaced by $\left(\frac{1}{2} \sqrt{g^{2}+g^{\prime 2}}\right)^{2}$ [51] [52], where the couplings $g, g^{\prime}$ are determined by $e^{2} / g^{2}=\sin ^{2} \theta_{\mathrm{W}}$ and $e^{2} / g^{\prime 2}=\cos ^{2} \theta_{\mathrm{W}}$ from the electroweak mixing angle $\theta_{\mathrm{W}}$. To determine the neutrino energy scale $\Lambda_{v}$ we may suggest the misalignment factor $\sin \zeta$ to enter a trailing

$$
2 \pi \Lambda_{v}=\left(\frac{1}{2} \sqrt{g^{2}+g^{\prime 2}}\right)^{2} \Lambda_{\mathrm{e}} \sin \zeta
$$

analogous to (50) with the Higgs vacuum expectation value $\varphi_{0}$ substituted by a residual electronic scale $\Lambda_{\mathrm{e}} \sin \zeta$. From (60) we get for the neutrino mass

$$
m_{v} c^{2}=\mathrm{E}_{v} \Lambda_{v}=\mathrm{E}_{v} \frac{\left(\frac{1}{2} \sqrt{g^{2}+g^{\prime 2}}\right)^{2}}{2 \pi} \Lambda_{\mathrm{e}} \sin \zeta .
$$

Using (47) for the misalignment $\sin \zeta$, we have

$$
m_{v} c^{2}=\mathrm{E}_{v} \frac{\left(\frac{1}{2} \sqrt{g^{2}+g^{\prime 2}}\right)^{2}}{2 \pi} \frac{\Lambda_{\mathrm{e}}^{2}}{\Lambda} .
$$

The strong scale $\Lambda=\hbar c / a=\hbar c /\left(r_{\mathrm{e}} / \pi\right)$ contains the classical electron radius $r_{\mathrm{e}}=e^{2} /\left(4 \pi \epsilon_{0} m_{\mathrm{e}} c^{2}\right)$ and can therefore be expressed as

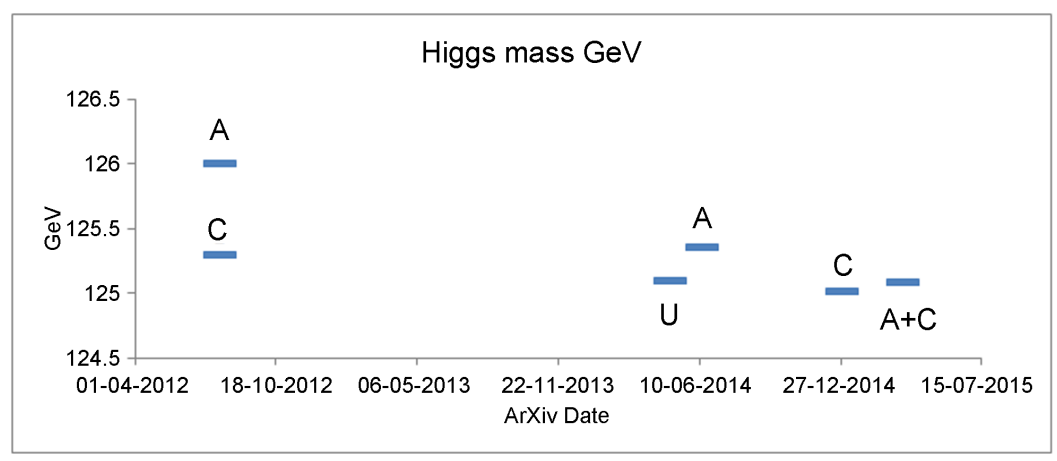

Figure 6. The chronology of experimental higgs mass values from the ATLAS collaboration (A) [47] [49] and the CMS collaboration (C) [48] [50] compared with our calculation $(\mathrm{U})$ [45]. The last result shown is from the combined data by ATLAS and CMS at LHC run $1(\mathrm{~A}+\mathrm{C})$ [46]. 


$$
\Lambda=\frac{\pi}{\alpha} m_{\mathrm{e}} c^{2}
$$

Inserting (63) in (62), using the identity

$$
\begin{aligned}
\left(\frac{1}{2} \sqrt{g^{2}+g^{\prime 2}}\right)^{2} & =\frac{1}{4}\left(\frac{e^{2}}{\sin ^{2} \theta_{\mathrm{W}}}+\frac{e^{2}}{\cos ^{2} \theta_{\mathrm{W}}}\right) \frac{1}{4 \pi \epsilon_{0} \hbar c} \\
& =\frac{1}{4} \frac{\alpha}{\sin ^{2} \theta_{\mathrm{W}} \cos ^{2} \theta_{\mathrm{W}}}
\end{aligned}
$$

and exploiting (10), we get

$$
\frac{m_{\bar{v}}}{m_{\mathrm{e}}}=\frac{1}{\pi \mathrm{E}_{\mathrm{e}}} \frac{\alpha_{\mathrm{z}}}{8 \sin ^{2} \theta_{\mathrm{W}} \cos ^{2} \theta_{\mathrm{W}}} \frac{\alpha_{\mathrm{e}}}{\pi} .
$$

This yields $m_{\bar{v}} c^{2}=0.9165(4) \mathrm{eV}$. The uncertainty on $\mathrm{E}_{\mathrm{e}}$ is estimated as $\mathrm{E}_{\mathrm{e}}=2.2655(5)$, i.e. of the order of the uncertainty on $\alpha_{\mathrm{z}}^{-1}$ corresponding to $0.022 \%$.

\section{Neutrino Mass Scenario I}

Instead of the second order misalignment in the Higgs mechanism leading to the result $m_{\bar{v}} c^{2}=0.917 \mathrm{eV}$ in (65) from (60), we look in the present section for a first order misalignment but with a different length scale set by the proton and electron charges in space, i.e. the Bohr radius.

Consider the classical electromagnetic field $A_{\mu}(x)$ for a proton at rest [53]

$$
A_{\mu}(x)=(\phi(x), 0,0,0)
$$

where $\phi(x)=\frac{e}{4 \pi \epsilon_{0}} \frac{1}{|x|}$ yields the Coulomb potential energy

$$
V(x)=\frac{e^{2}}{4 \pi \epsilon_{0}} \frac{1}{|x|}=\alpha_{\mathrm{e}} \frac{\hbar c}{|\boldsymbol{x}|}
$$

for an electric charge $e$ at a distance $|\boldsymbol{x}|$ from the proton. Here we separated out the fine structure constant $\alpha_{\mathrm{e}}=e^{2} /\left(4 \pi \epsilon_{0} \hbar c\right)$ in order to enter into a quantum regime. We reinterpret $\alpha$ as a dimensionless coupling constant in quantum field theory. To set up the trailing in this scenario we make the substitutions

$$
\frac{\hbar c}{|x|} \rightarrow \varphi_{\mathrm{B}} \quad \text { and } \quad V(x) \rightarrow \alpha \varphi_{\mathrm{B}}
$$

to get $\varphi_{\mathrm{B}}$ of dimension energy at a characteristic length scale of the Bohr radius $a_{\infty}$

$$
|x|=a_{\infty}=4 \pi \epsilon_{0} \hbar^{2} /\left(e^{2} m_{\mathrm{e}}\right)=r_{\mathrm{e}} \alpha_{\mathrm{e}}^{-2} .
$$

We use the Bohr radius [2] as a characteristic scale by imagining the creation of the anti-electron-neutrino to happen at a length scale given by the electromagnetic interaction in space between the electric charges of the proton and electron created during the neutron decay. This assumption expresses the fact that all three particles $p, e$ and $\bar{v}_{\mathrm{e}}$ are created simultaneously in the neutron 
decay. Thus, the proton and the electron charges (together with their masses) by defining the scale of the hydrogen atom corresponding to $a_{\infty}$-sets the scene for the projection of the intrinsic dynamics of the anti-electron-neutrino. If we accept (69) as a reasonable length scale, we get a trailing

$$
2 \pi \Lambda_{v}=\left(\frac{1}{2} \sqrt{g^{2}+g^{\prime 2}}\right)^{2} \frac{\hbar c}{a_{\infty}} \sin \zeta
$$

where the charged current coupling $\alpha$ is substituted by the neutral charge

$$
\begin{aligned}
& \text { coupling }(f / 2)^{2}=\left(\frac{1}{2} \sqrt{g^{2}+g^{\prime 2}}\right)^{2}[51][52] \text { and } \\
& \qquad \varphi_{\mathrm{B}} \sin \zeta=\frac{\hbar c}{a_{\infty}} \sin \zeta
\end{aligned}
$$

is the residual energy scale for neutrino mass creation. Thus the minimum space action exchange behind (70) is

$$
h c=a_{\infty}\left(\frac{1}{2} \sqrt{g^{2}+g^{\prime 2}}\right)^{2} \varphi_{\mathrm{B}} \sin \zeta=\tau_{\mathrm{B}} c \Lambda_{v}
$$

Like in (37), the characteristic time $\tau_{\mathrm{B}}$ is determined by

$$
c \tau_{\mathrm{B}}=2 \pi a_{\infty}
$$

which inserted in (72) gives (70). From (70) and (10) follows

$$
\frac{m_{\bar{V}}}{m_{\mathrm{e}}}=\frac{\Lambda_{v}}{\Lambda_{\mathrm{e}}}=\frac{\alpha_{\mathrm{z}}}{8 \sin ^{2} \theta_{\mathrm{W}} \cos ^{2} \theta_{\mathrm{W}}}\left(\frac{\alpha_{\mathrm{e}}}{\pi}\right)^{2}
$$

as stated in (2). With $\alpha_{\mathrm{Z}}^{-1}=127.940(14), \sin ^{2} \theta_{\mathrm{W}}=0.23126(5)$, $\alpha_{\mathrm{e}}^{-1}=137.035999074(44)$ and $m_{\mathrm{e}} c^{2}=0.510998928(11) \mathrm{MeV}$ [2] this yields $m_{\bar{V}} c^{2}=15.152(4) \mathrm{meV}$ as already mentioned. To observe a neutrino mass this small would require an improvement by one and a half order of magnitude from the sensitivity of the KATRIN experiment [8] but lies close to the sensitivity of the CRES technique [17] prospected in Project 8.

\section{Discussion}

The second scenario with $m_{\bar{v}} c^{2}=0.9165(4) \mathrm{eV}$ combined with the observed mass square differences (4) from neutrino oscillations means nearly degenerate neutrino mass eigenstates of the order of $1 \mathrm{eV}$. This is in conflict with cosmological constraints [16] as mentioned in the introduction. For the first scenario we follow the interpretation implied by the Project 8 collaboration, that the lowest mass eigenstate should be of the order of $\sqrt{\left|\Delta m_{21}^{2}\right|}$. We infer in normal hierarchy from (2) and (4)

$$
\begin{gathered}
m_{1} c^{2}=15.152 \pm 0.004 \mathrm{meV} \\
m_{2}^{2}=\Delta m_{21}^{2}+m_{1}^{2} \rightarrow m_{2} c^{2}=17.5 \pm 0.1 \mathrm{meV} \\
m_{3}^{2}=\Delta m_{32}^{2}+m_{2}^{2} \rightarrow m_{3} c^{2}=52.4 \pm 0.6 \mathrm{meV} .
\end{gathered}
$$

This leads to a sum-total

$$
\Sigma=m_{1} c^{2}+m_{2} c^{2}+m_{3} c^{2}=85.0 \pm 0.6 \mathrm{meV} .
$$


Using (6) with $U_{e 1}=\cos \theta_{12} \cos \theta_{13}, U_{e 2}=\sin \theta_{12} \cos \theta_{13} \quad$ and

$U_{e 3}=\sin \theta_{13} e^{-i \delta} \quad$ where $\sin ^{2} \theta_{12}=0.308 \pm 0.017, \sin ^{2} \theta_{13}=0.0234_{-0.0019}^{+0.0020} \quad$ and $\delta / \pi=1.39_{-0.27}^{+0.38} \quad$ [54] we infer

$$
m_{\beta} c^{2} \approx 17.6 \pm 0.2 \mathrm{meV}
$$

in accordance with what can be read off from figure 10 in ref. [55] correlating beta neutrino mass with cosmological constraints for the sum-total mass in (76) and in agreement with the disfavouring of inverted hierarchy in recent results from the NOvA neutrino oscillation experiment [56]. Note that the complex phase $\delta$ cancels out in the norm square of the matrix elements $U_{e j}$ in (6) as does possible Majorana phases.

\section{Conclusion}

We have investigated two possible scale scenarios for neutrino mass generation. Both scales relate to an intrinsic conception of the origin of the Higgs potential. This conception leads to slight discrepancies from standard model expectations in the quadric and triple Higgs self-couplings by having the $d$ to $u$ quark mixing matrix element as a factor in the electroweak energy scale $v$ derived from neutron to proton decay. The foundation we have suggested is that of exchange of minimum quanta of action which can be used without knowing the detailed mechanisms behind the exchange between various degrees of freedom. We look forward to future accelerator experiments to test the Higgs self-couplings and to results from KATRIN and Project 8 to determine or constrain neutrino masses and possibly clarify the mechanisms behind neutrino mass generation.

\section{Acknowledgements}

I thank my colleagues H. G. Bohr and M. S. Jensen for co-work on the Higgs mass and H. G. Bohr for co-work on the pion mass. I thank both for showing interest in the intrinsic viewpoint and I thank the Technical University of Denmark for an inspiring working environment.

\section{References}

[1] Nakamura, K. and Petrov, S.T. (2014) Neutrino Mass, Mixing and Oscillations. In: Olive, K.A., et al. (2014) Chinese Physics C, 38, Article ID: 090001, 235-258.

[2] Olive, K.A., et al. (2014) Chinese Physics C, 38, Article ID: 090001.

[3] Cleveland, B.T., Daily, T., Davis, Jr., R., Distel, J.R., Lande, K., Lee, C.K., Wildenhain, P.S. and Ullman, J. (1988) The Astrophysical Journal, 496, 505. https://doi.org/10.1086/305343

[4] Abe, K., et al. (2013) Physical Review Letters, 110, Article ID: 181802. https://doi.org/10.1103/PhysRevLett.110.181802

[5] Pastore A. (2013) Recent Results of the OPERA Neutrino Experiment. The EPS HEP 2013 Conference, Stockholm, 18-24 July 2013.

[6] Adamson, P., et al. (2013) Physical Review Letters, 110, Article ID: 171801.

[7] Abe, K., et al. (2014) Physical Review Letters, 112, Article ID: 061802. 
[8] Thümmler, T., et al. (2010) Nuclear Physics B-Proceedings Supplements, 229-232, 146-151. https://doi.org/10.1016/j.nuclphysbps.2012.09.024

[9] Fränkle, F. (2015) Status of the Neutrino Experiment KATRIN and Project 8. Proceedings of the European Physical Society Conference on High Energy Physics, Vienna, 22-29 July 2015, PoS(EPS-HEP2015)084.

[10] Dragoun, O. and Vénos, D. (2016) Open Physics Journal, 3, 73-113.

[11] Englert, F. and Brout, R. (1964) Physical Review Letters, 13, 321-323. https://doi.org/10.1103/PhysRevLett.13.321

[12] Higgs, P.W. (1964) Physics Letters, 12, 132-133. https://doi.org/10.1016/0031-9163(64)91136-9

[13] Higgs, P.W. (1964) Physical Review Letters, 13, 508-509. https://doi.org/10.1103/PhysRevLett.13.508

[14] Guralnik, G.S., Hagen, C.R. and Kibble, T.W.B. (1964) Physical Review Letters, 13, 585-587. https://doi.org/10.1103/PhysRevLett.13.585

[15] Higgs, P.W. (1966) Physical Review, 145, 1156-1163. https://doi.org/10.1103/PhysRev.145.1156

[16] Olive, K.A., et al. (2014) Chinese Physics C, 38, Article ID: 090001, 692.

[17] Esfahani, A.A., et al. (2017) Journal of Physics G: Nuclear and Particle Physics, 44, Article ID: 054004, 16 p.

[18] Olive, K.A., et al. (2014) Chinese Physics C, 38, Article ID: 090001, 235.

[19] Olive, K.A., et al. (2014) Chinese Physics C, 38, Article ID: 090001, 689.

[20] Olive, K.A., et al. (2014) Chinese Physics C, 38, Article ID: 090001, 253.

[21] Weyl, H. (1997) The Classical Groups-Their Invariants and Representations. 2nd Edition, Princeton University Press, Princeton, 197.

[22] Milnor, J. (1963) Annals of Mathematics Studies, 51, 1.

[23] Dowker, J.S. (1971) Annals of Physics, 62, 361-3822. https://doi.org/10.1016/0003-4916(71)90096-0

[24] Trinhammer, O.L. and Olafsson, G. (1999) The Full Laplace-Beltrami Operator on $\mathrm{U}(\mathrm{N})$ and $\mathrm{SU}(\mathrm{N})$. arXiv:9901002v2 [math-ph]

[25] Ashcroft, N.W. and Mermin, N.D. (1976) Solid State Physics. Holt, Rinehart and Winston, New York, 160.

[26] Slater, J.C. (1929) Physical Review, 34, 1293. https://doi.org/10.1103/PhysRev.34.1293

[27] Bruun Nielsen, H. (1997) Technical University of Denmark. [Private Communication].

[28] Trinhammer, O.L. (2012) Neutron to Proton Mass Difference, Parton Distribution Functions and Baryon Resonances from Dynamics on the Lie Group $\mathrm{u}(3)$. arXiv:1109.4732v3 [hep-th]

[29] Kogut, J.B. and Susskind, L. (1974) Physical Review D, 10, 395.

[30] Trinhammer, O.L. (2013) Europhysics Letters, 102, Article ID: 42002.

[31] Heisenberg, W. (1938) Annalen der Physik, 32, 20-33. https://doi.org/10.1002/andp.19384240105

[32] Landau, L.D. and Lifshitz, E.M. (2005) The Classical Theory of Fields, Course of Theoretical Physics Vol. 2. 4th Edition, Elsevier Butterworth-Heinemann, Oxford, 97.

[33] Trinhammer, O.L., Bohr, H.G. and Jensen, M.S. (2015) International Journal of 
Modern Physics, A30, Article ID: 1550078.

[34] Bohr, Aa. and Mottelson, B.R. (1969) Nuclear Structure, Vol. 1. W. A. Benjamin, New York, Amsterdam, 87.

[35] Bohr, Aa. and Mottelson, B.R. (1969) Nuclear Structure, Vol. 1. W. A. Benjamin, New York, Amsterdam, 78.

[36] Trinhammer, O.L. (2017) Intrinsic Quantum Mechanics I. Foundation and Applications to Particle Physics: $\left(m_{n}-m_{p}\right) / m_{p}, N \mathrm{~s}, \Delta \mathrm{s}, P_{c}^{0} \mathrm{~s}, P D F \mathrm{~s}, g_{1}^{P}, \mu_{p}$. Neutral Pentaquarks, Proton Spin Structure and Magnetic Moment. ResearchGate.

[37] Wilson, K.G. (1974) Physical Review D, 10, 2445-2459. https://doi.org/10.1103/PhysRevD.10.2445

[38] Manton, N.S. (1980) Physics Letters B, 96, 328-330. https://doi.org/10.1016/0370-2693(80)90778-9

[39] Jacobsen, H.P. (1997) Private Communication. Department of Mathematics, University of Copenhagen, Denmark.

[40] Trinhammer, O.L., Bohr, H.G. and Jensen, M.S. (2015) A Higgs at $125.1 \mathrm{GeV}$ and Baryon Mass Spectre Derived from a Common U(3) Lie Group Framework. Proceedings of the European Physical Society Conference on High Energy Physics, Vienna, 22-29 July 2015, PoS(EPS-HEP2015)097.

[41] Trinhammer, O.L. and Bohr, H.G. (2017) Intrinsic Quantum Mechanics III. Derivation of Pion Mass and Decay Constant. ResearchGate.

[42] Gupta, R.S., Rzehak, H. and Wells, J.D. (2013) Physical Review D, 88, Article ID: 055024. https://doi.org/10.1103/PhysRevD.88.055024

[43] Olive, K.A., et al. (2014) Chinese Physics C, 38, Article ID: 090001, 163, 109.

[44] Weinberg, S. (1995/2012) The Quantum Theory of Fields-Modern Applications, Vol II. Cambridge University Press, Cambridge, 185. https://doi.org/10.1017/CBO9781139644167

[45] Trinhammer, O.L. (2014) A Higgs Mass at $125 \mathrm{GeV}$ Calculated from Neutron to Proton Decay in a u(3) Lie Group Hamiltonian Framework. arXiv: 1302.1779v2 [hep-ph]

[46] ATLAS, CMS Collaborations (2015) Physical Review Letters, 114, Article ID: 191803.

[47] ATLAS Collaboration (2012) Physics Letters B, 716, 1. https://doi.org/10.1016/j.physletb.2012.08.020

[48] CMS Collaboration (2012) Physics Letters B, 716, 30. https://doi.org/10.1016/j.physletb.2012.08.021

[49] ATLAS Collaboration (2014) Physical Review D, 90, Article ID: 052004.

[50] CMS Collaboration (2014) The European Physical Journal C, 75, 212.

[51] Scheck, F. (1996/2012) Electroweak and Strong Interactions. Phenomenology, Concepts, Models. 3rd Edition, Graduate Texts in Physics, Springer-Verlag, Berlin, Heidelberg, Germany, 212. https://doi.org/10.1007/978-3-662-03245-9

[52] Walecka, J.D. (2004/2013) Theoretical Nuclear and Subnuclear Physics. 2nd Edition, Imperial College Press, World Scientic, London, Singapore, 440. https://doi.org/10.1142/5500

[53] Scheck, F. (1996/2012) Electroweak and Strong Interactions. Phenomenology, Concepts, Models. 3rd Edition, Graduate Texts in Physics, Springer-Verlag, Berlin, Heidelberg, Germany, 61. https://doi.org/10.1007/978-3-662-03245-9

[54] Olive, K.A., et al. (2014) Chinese Physics C, 38, Article ID: 090001, 252. 
[55] Capozzi, F., Valentino, E.D., Lisi, E., Marrone, A., Melchiorri, A. and Palazzo, A. (2017) Global Constraints on Absolute Neutrino Masses and Their Ordering. arXiv: 1703.04471v1 [hep-ph]

[56] Adamson, P., et al. (2017) Constraints on Oscillation Parameters from $v_{\mu}$ Disappearance in NOvA. arXiv: 1703.03328v1, [hep-ex]

Submit or recommend next manuscript to SCIRP and we will provide best service for you:

Accepting pre-submission inquiries through Email, Facebook, LinkedIn, Twitter, etc. A wide selection of journals (inclusive of 9 subjects, more than 200 journals) Providing 24-hour high-quality service User-friendly online submission system Fair and swift peer-review system Efficient typesetting and proofreading procedure Display of the result of downloads and visits, as well as the number of cited articles Maximum dissemination of your research work

Submit your manuscript at: http://papersubmission.scirp.org/ Or contact jmp@scirp.org 